\title{
Roper state from overlap fermions
}

\author{
Mingyang Sun, ${ }^{1}$ Ying Chen, ${ }^{2}$ Gen Wang $\odot,{ }^{1}$ Andrei Alexandru, ${ }^{3}$ Shao-Jing Dong, ${ }^{1}$ Terrence Draper, ${ }^{1}$ Jacob Fallica, ${ }^{1}$ \\ Ming Gong, ${ }^{2}$ Frank X. Lee, ${ }^{3}$ Anyi Li, ${ }^{4}$ Jian Liang $\odot,{ }^{1}$ Keh-Fei Liu $\odot,{ }^{1,}$ Nilmani Mathur, ${ }^{5}$ and Yi-Bo Yang $\oplus^{6}$ \\ $(\chi \mathrm{QCD}$ Collaboration) \\ ${ }^{1}$ Department of Physics and Astronomy, University of Kentucky, Lexington, Kentucky 40506, USA \\ ${ }^{2}$ Institute of High Energy Physics, Chinese Academy of Sciences, Beijing 100049, China \\ ${ }^{3}$ Department of Physics, The George Washington University, Washington, D.C. 20052, USA \\ ${ }^{4}$ Institute for Nuclear Theory, University of Washington, Seattle, 98195, USA \\ ${ }^{5}$ Department of Theoretical Physics, Tata Institute of Fundamental Research, Mumbai 400005, India \\ ${ }^{6}$ Institute of Theoretical Physics, Chinese Academy of Sciences, Beijing 100190, China
}

(Received 1 November 2019; accepted 10 March 2020; published 30 March 2020)

The Roper state is extracted with valence overlap fermions on a $2+1$-flavor domain-wall fermion lattice (spacing $a=0.114 \mathrm{fm}$ and $m_{\pi}=330 \mathrm{MeV}$ ) using both the sequential empirical Bayes (SEB) method and the variational method. The results are consistent, provided that a large smearing-size interpolation operator is included in the variational calculation to have better overlap with the lowest radial excitation. The SEB and variational calculation with large smearing size are also carried out for an anisotropic clover lattice with similar parameters (spatial lattice spacing $a_{s}=0.12 \mathrm{fm}$ and pion mass $m_{\pi}=396 \mathrm{MeV}$ ) and obtain consistent results. However, these calculations with clover fermions give a Roper mass of $m_{R}=1.92(6) \mathrm{GeV}$, while the same approach with overlap fermions finds the Roper $\approx 280 \mathrm{MeV}$ lower, at $m_{R}=1.64(9) \mathrm{GeV}$, for identical valence pion mass. The fact that the prediction of the Roper state by overlap fermions is consistently lower than those of clover fermions, chirally improved fermions, and twisted-mass fermions over a wide range of pion masses has been dubbed a "Roper puzzle." To understand the origin of this difference, we study the hairpin $Z$-diagram in the isovector scalar meson $\left(a_{0}\right)$ correlator in the quenched approximation. The lack of quark loops in the quenched approximation turns the $a_{0}$ correlator negative; giving rise to a ghost "would-be" $\eta \pi$ state. Comparing the $a_{0}$ correlators for valence clover and overlap fermions, at a valence pion mass of $290 \mathrm{MeV}$, on three quenched Wilson-gauge lattices, we find that the spectral weight of the ghost state with clover fermions is smaller than that of the overlap at $a=0.12 \mathrm{fm}$ and $0.09 \mathrm{fm}$ - the ratios of the Wilson ghost-state magnitudes (correlator minima) are about half of those of overlap-whereas, the whole $a_{0}$ correlators of clover and overlap at $a=0.06$ fm coincide within errors. This suggests that chiral symmetry is restored for clover at $a \leq 0.06 \mathrm{fm}$ and that the Roper mass should agree between clover and overlap fermions toward the continuum limit. We conclude that the present work supports a resolution of the "Roper puzzle" due to Z-graph type chiral dynamics. This entails coupling to higher components in the Fock space (e.g., $N \pi, N \pi \pi$ states) to induce the effective flavor-spin interaction between quarks as prescribed in the chiral quark model, resulting in the parity-reversal pattern as observed in the experimental excited states of $N, \Delta$ and $\Lambda$.

DOI: 10.1103/PhysRevD.101.054511

\section{INTRODUCTION}

The nature of the lowest nucleon excited state, the Roper $\mathrm{N}(1440)$ which appears in the $\pi N$ scattering in the $P_{11}$

*liu@g.uky.edu

Published by the American Physical Society under the terms of the Creative Commons Attribution 4.0 International license. Further distribution of this work must maintain attribution to the author(s) and the published article's title, journal citation, and DOI. Funded by SCOAP ${ }^{3}$. channel at about $1370 \mathrm{MeV}$ with a width of about $175 \mathrm{MeV}$, has been a controversial and intriguing subject since its discovery. First of all, it is rather unusual to have the first positive-parity excited state lower than the negative-parity excited state, which is the $N^{\frac{1}{2}^{-}}(1535)$ at $1510 \mathrm{MeV}$ in the $\pi N S_{11}$ scattering channel. This is contrary to the excitation pattern in the meson sectors with either light or heavy quarks. This parity reversal has caused problems for the otherwise successful quark models based on $S U(6)$ symmetry with color-spin interaction between 
the quarks, which cannot accommodate such a pattern. Realistic potential calculations with linear and Coulomb potentials [1] and the relativistic quark model [2,3] all predict the Roper to be $\sim 100-200 \mathrm{MeV}$ above the experimental value, and above the negative parity state. On the other hand, the pattern of parity reversal was readily obtained in a chiral soliton model, the Skyrme model, via the small oscillation approximation to $\pi N$ scattering. Although the first calculations [4,5] of the original Skyrme model gave rise to a breathing mode which is $\sim 200 \mathrm{MeV}$ lower than the Roper resonance, it was shown later [6] that the introduction of the sixth-order term, which is the zerorange approximation for the $\omega$ meson coupling, changes the compression modulus and yields better agreement with experiment for both the mass and width in $\pi N$ scattering.

Since the quark potential model is based on $S U(6)$ symmetry with residual color-spin interaction between the quarks, whereas the chiral soliton model is based on spontaneous broken chiral symmetry, their distinct predictions on the ordering of the positive- and negative-parity excited states are most likely a reflection of different dynamics derived from their respective symmetries. This possibility has prompted the suggestion [7] that the parity reversal in the excited nucleon and $\Delta$, in contrast to that in the excited $\Lambda$ spectrum, is an indication that the interquark interaction of the light quarks is mainly of the flavor-spin nature rather than the color-spin nature (e.g., one-gluon exchange type). This suggestion is supported in the lattice QCD study of "valence QCD" [8] which finds that the hyperfine splitting between the nucleon and $\Delta$ is greatly diminished when the $Z$-graphs in the quark propagator are eliminated by modifying the fermion action to prevent the quarks from propagating backwards in time. This is an indication that the color-magnetic interaction is not the primary source of the interquark spin-spin interaction for light quarks. (The color-magnetic part, being spatial in origin, is unaffected by the truncation of Z-graphs in Valence QCD, which only affects the temporal part.) Yet, it is consistent with the flavor-spin interaction being generated by the quark $Z$-graph in the Goldstone-bosonexchange picture. This picture is enhanced by the extensive dynamical coupled- channel (DCC) model analysis carried out by the excited baryon analysis center [EBAC] at JLab [9-11]. This DCC Hamiltonian approach involves a nucleon core and the meson-baryon reactions in the $\pi N$, $\eta N$, and $\pi \pi N$ channels which fits 22,348 independent data points, representing the complete array of partial waves below $2 \mathrm{GeV}$. In the $\pi N P_{11}$ partial wave, it is found that starting with a bare state at $1.763 \mathrm{GeV}$, two resonances merge into the Roper resonance. They are at $1357 \mathrm{MeV}$ with $\Gamma=152 \mathrm{MeV}$ and $1364 \mathrm{MeV}$ with $\Gamma=210 \mathrm{MeV}$. There is a third resonance at $1820 \mathrm{MeV}$ with $\Gamma=496 \mathrm{MeV}$ which may correspond to $N^{*}(1710)$. It is interesting to note that the meson-baryon coupling brings down the bare state by $\approx 400 \mathrm{MeV}$, to the Roper mass. The failure of the $S U(6)$ quark model to delineate the Roper and its photoproduction has also prompted the speculation that the Roper resonance may be a hybrid state with excited glue [12-14] or a $q q q q \bar{q}$ five-quark state $[15,16]$. Thus, unraveling the nature of Roper resonance has direct bearing on our understanding of the quark structure and chiral dynamics of baryons, which is one of the primary missions at experimental facilities such as at Jefferson Lab [17]. At the moment, the nature of Roper and why it is lower than the quark model prediction as the radial excitation of the nucleon is unsettled.

Lattice QCD, being a first-principles approach, is regarded as the most desirable tool to adjudicate the theoretical controversy surrounding the issue and to discern the nature of Roper. However, lattice calculations of the Roper state are also shrouded in a puzzle which we shall address and sort out in this manuscript. Studying the ghost would-be $\eta \pi$ state in the quenched approximation has paved the way to better understand the chiral dynamics and the origin of the difference due to fermion actions with and without chiral symmetry at finite lattice spacing.

This manuscript is organized as follows. The status of lattice calculations of Roper are reported in Sec. II where the discrepancy between results from Wilson-type fermions and those from the overlap fermions is pointed out. Sec. III will see calculations of both the SEB and variational methods for the clover and overlap fermions to check the consistency of the two approaches in obtaining the nucleon excited states. In an attempt to understand the puzzling difference of the Roper between the two fermion actions in terms of chiral dynamics, we calculate the ghost would-be- $\eta \pi$ state in the quenched approximation for the overlap and Wilson fermions in Sec. IV. This is a sensible and pertinent place to compare the coupling strength of single-hadron interpolating fields to two-hadron states for these fermion actions as a function of lattice spacing. We finish in Sec. V with a summary and conclusion.

\section{ROPER PUZZLE FROM LATTICE CALCULATIONS}

Lattice calculations of the positive-parity excitation of the nucleon started out with the quenched approximation. The first set of calculations used a nucleon interpolation field which does not have a nonrelativistic limit to project to the excited $1 / 2^{+}$state [18-22] and found it to be much higher than the Roper. Later, Bayesian methods $[23,24]$ and variational approaches with multiple interpolation operators [25-29] were introduced in the calculation of the excited state. The results of the calculations are plotted in Fig. 1 as a function of $m_{\pi}^{2}$ together with the corresponding nucleon masses. The nucleon masses from different calculations are all in agreement within errors. However, one distinct feature stands out, i.e., the excited $\frac{1}{2}^{+}$states do not approach the experimental Roper state at the physical pion point upon chiral extrapolation. The only exception is overlap fermions for which the Roper mass is consistently 


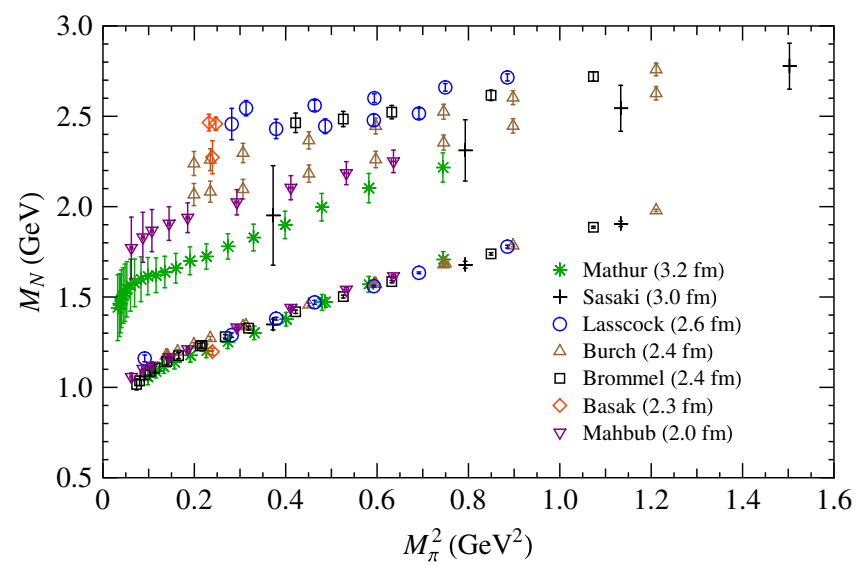

FIG. 1. Quenched results of masses of the nucleon ground state and first positive-parity excited state for a broad range of pion masses. The green stars are results obtained from the overlap fermion.

lower than in the other calculations at each value of $m_{\pi}^{2}$. The situation with the dynamical fermion calculations is basically a replication of that of the quenched approximation. As shown in Fig. 2, the variational calculations of $N_{f}=$ $2+1$ clover fermions from the CSSM Collaboration [30,31], an anisotropic lattice calculation from the JLab Collaboration [32], an $N_{f}=2$ clover and twisted-mass fermions studies from the Cypress group [33], and a chirally improved fermion study from the BGR Collaboration (N.B. The result is from chiral extrapolation to the physical pion mass) [34], all yield much higher Roper masses at the same $m_{\pi}^{2}$ than those of the overlap calculation $[35,36]$ which uses the SEB method. Especially near the physical pion mass, the results from the clover fermion [31,33], are $\sim 400 \mathrm{MeV}$ above the experimental Roper mass at $1370 \mathrm{MeV}$. The twisted mass calculation from the Cypress group [33], with large smearing sources in the variational approach, gives lower Roper masses than those of the clover fermion and is closer to that of the overlap fermion at $m_{\pi} \sim 300 \mathrm{MeV}$, but higher at other pion masses, especially at $m_{\pi}=270 \mathrm{MeV}$. Recently, a calculation was made that used both $q^{3}$ and $q^{4} \bar{q}$ (interpolation field in the form of $N \pi$ and $N \sigma$ ) operators on a $2+1$-flavor clover fermion lattice with $a=0.0907 \mathrm{fm}$ and $m_{\pi}=156 \mathrm{MeV}$ to check if the Roper state could be produced through the coupling of higher Fock space [37]. No Roper state was found below $1.65 \mathrm{GeV}$ in this coupledchannel scattering calculation. The same conclusion has been drawn from a similar calculation on the same clover fermion lattice except at $m_{\pi}=411 \mathrm{MeV}$ [38].

In contrast to the clover and twisted-mass results, it has been shown $[35,36]$ in Fig. 2 that the first positive-parity excited nucleon state from overlap fermions with the SEB method is consistently $\sim 500 \mathrm{MeV}$ above the corresponding nucleon mass in the pion mass range up to $\sim 580 \mathrm{MeV}$. This behavior is consistent with the typical size of the radial excitation of $\sim 500 \mathrm{MeV}$ for the nucleon, $\Delta, \Lambda$ and $\Sigma$ as well as heavy quarkoniums. The overlap calculation with the SEB method is based on $2+1$-flavor domain-wall fermion configurations on the $24^{3} \times 64$ lattice with $a=$ $0.114 \mathrm{fm}$ and sea pion mass at $330 \mathrm{MeV}$. Multiple overlap valence quark masses are used in this partially-quenched calculation to cover the valence pion mass range from $260 \mathrm{MeV}$ to $580 \mathrm{MeV}$. We fit the available data for different quark masses with the form

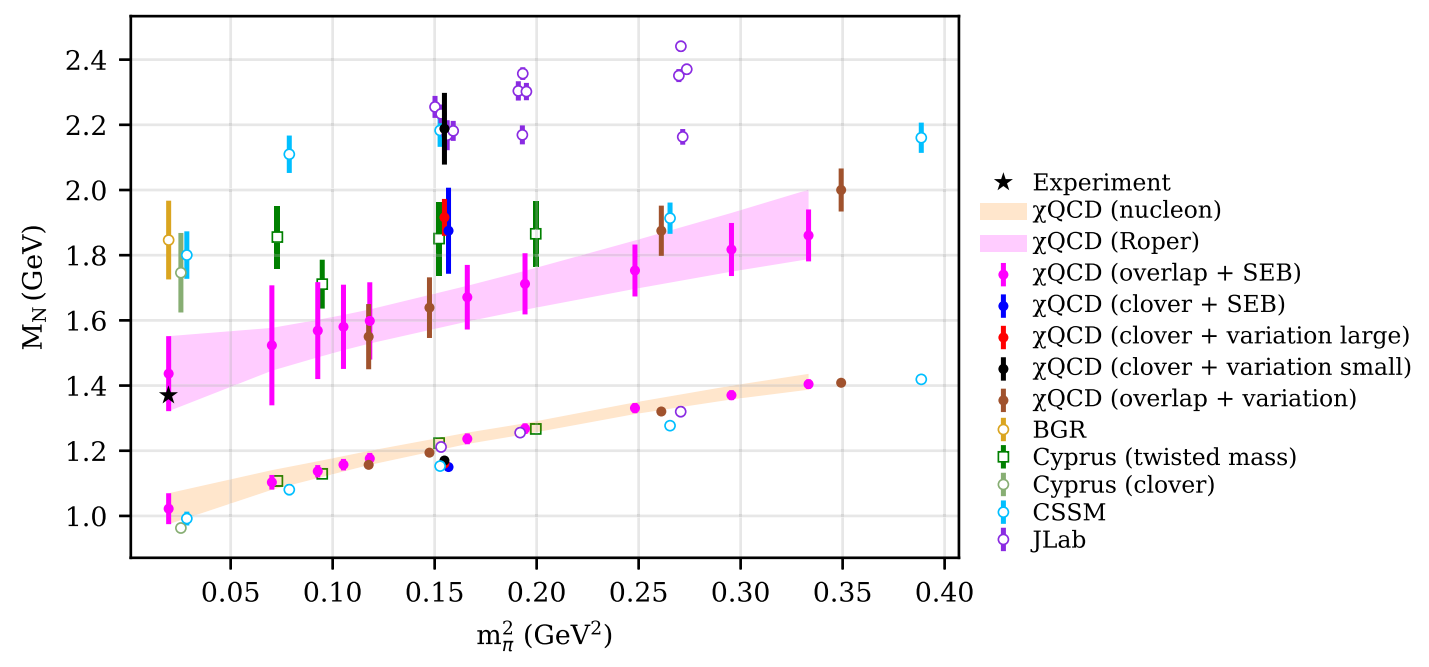

FIG. 2. Results with dynamical fermion configurations for the masses of the nucleon ground state and its first positive-parity excited state across several pion masses. The magenta points and band are results obtained from valence overlap fermions on the domain-wall sea with the SEB method. The brown points are from the variational results on the same lattice. The blue point is the SEB result on the JLab clover configurations. The red/black point is the variational result on the JLab clover configurations with large/small interpolation fields. Also listed are results from BGR Collaboration [34], Cyprus with twisted mass and clover fermions [33], CSSM with clover fermions [30,31], and JLab with clover fermions on an anisotropic lattice [32]. 


$$
M_{N, R}=M_{N, R}(0)+c_{2(N, R)} m_{\pi, v v}^{2}+c_{3(N, R)} m_{\pi, v s}^{3}
$$

where $m_{\pi, v v}$ is the valence pion mass. The $m_{\pi, v s}^{3}$ term is introduced to reflect the effects of partial quenching between the valence and the sea quark mass. The partially quenched pion mass $m_{\pi, v s}$ is made of a combination of the valence quark/antiquark and the sea antiquark/quark with the relation $m_{\pi, v s}^{2}=1 / 2\left(m_{\pi, v v}^{2}+m_{\pi, s s}^{2}\right)+\Delta_{\text {mix }} a^{2}$, where $\Delta_{\text {mix }}$ is a low-energy constant for the mixed action. Since the overlap fermion action and the domain wall fermion action are close to each other, we obtain a small $\Delta_{\text {mix }}$ [39]. The value $\Delta_{\text {mix }}=0.030(6)(5) \mathrm{GeV}^{4}$ we obtained is calculated on lattices with two lattice spacings $(0.014 \mathrm{fm}$ and $0.085 \mathrm{fm}$ ) and is an order of magnitude smaller than those of DWF on staggered and overlap on clover [39]. It gives a shift of only $16 \mathrm{MeV}$ to the valence pion mass at $300 \mathrm{MeV}$ for the lattice we use in this work. At the physical pion limit, we obtain $M_{N}=0.999$ (46) $\mathrm{GeV}$ and $M_{R}=1.40(11) \mathrm{GeV}$, in good agreement with experiment. In Fig. 2, the data points are from the SEB fit to the results from the full gauge ensemble and the error bars are obtained directly from the fit. We also carry out a singleelimination jackknife analysis by performing the SEB fit to each jackknive ensemble, and treat the jackknife error of each data point as the statistical error to build the covariance error matrix. Based on this jackknife analysis, the chiral extrapolation was carried out using Eq. (1) in the manuscript, which is illustrated in the figure by the error band.

Based on this observation, it has been speculated that the difference could be due to the fact that the overlap fermion is a chiral fermion which induces different dynamics for the excited nucleon. However, there is a caveat in that the overlap calculation employed the SEB method [40]. This method, although successfully applied in calculating $S_{11}(1535)$ [24], $a_{0}(1450)$ and $\sigma(600)$ [41], and the radially excited $1 P$ charmonium state [42], has not been tested by other groups. The variational approach is considered a robust and trustworthy algorithm for excited-state calculation. Thus, there is no consensus on this puzzle and one needs to sort out the issue regarding different algorithms first.

\section{VARIATIONAL AND SEQUENTIAL EMPIRICAL BAYES CALCULATIONS}

The only sensible way to check if the discrepancy between the overlap fermion results from the SEB method and those of other fermions from the variational method is due to the different algorithmic approaches is to apply both methods for the same fermion action and on the same lattice to see if they produce consistent results. To this end, we choose to examine two cases: an ensemble using clover fermions from JLab $[43,44]$ and another using valence overlap fermions on a domain-wall sea generated by $\mathrm{RBC} /$ UKQCD [44,45]. The parameters of the clover [43] and the domain-wall $[44,45]$ lattices are tabulated in Table I.
TABLE I. Lattice parameters of the clover and domain-wall fermion configurations including the lattice size, spatial lattice spacing $a_{s}$, the anisotropic factor $\xi$, the pion mass from the light sea quark mass, and the number of gauge configurations $N_{\text {conf }}$ are listed.

\begin{tabular}{lllllll}
\hline \hline Fermion & $L^{3} \times T$ & $a_{s}(\mathrm{fm})$ & $\xi$ & $\mathrm{L}(\mathrm{fm})$ & $m_{\pi}(\mathrm{MeV})$ & $N_{\text {conf }}$ \\
\hline Clover & $24^{3} \times 128$ & 0.123 & 3.5 & 2.95 & 396 & 761 \\
Domain wall & $24^{3} \times 64$ & 0.114 & 1 & 2.74 & 330 & 203 \\
\hline \hline
\end{tabular}

We first applied the SEB method on the clover configurations with the same clover fermion as used by the JLab group, except that we worked with a larger $24^{3} \times 128$ lattice instead of the $16^{3} \times 128$ lattice used to calculate Roper in Ref. [32] at the same quark mass. The result is plotted in Fig. 2. We see that the nucleon mass (blue point) is consistent with that from the JLab calculation [32], but the first positive-parity state from SEB (blue point) at 1.87 (13) $\mathrm{GeV}$ is lower than that of the JLab calculation at $\sim 2.20(10) \mathrm{GeV}$ by $\sim 300 \mathrm{MeV}$ with more than a $2.5 \sigma$ difference. To check if this difference is due to the fitting algorithms, we performed variational calculations with different gauge-invariant Gaussian smearing sizes for the nucleon source and sink.

Before addressing the variational calculation, it is worthwhile noticing that, to the extent that the Roper is the radially excited nucleon, its primary distinction from the nucleon is its radial wave function. This will affect the smearing size of the source and sink to be used in the variational calculation. To this end, we show in Fig. 3 the Roper and nucleon wave functions which were obtained by placing two $u$ quarks of the sink interpolation field for the proton at the same point and the $d$ quark at a distance $R$ away on a Coulomb gauge-fixed lattice. These were obtained from a quenched calculation with the overlap fermion on a $16^{3} \times 28$ lattice with $a=$ $0.2 \mathrm{fm}$ [46]. We note that for $m_{\pi}$ at both $633 \mathrm{MeV}$ and $438 \mathrm{MeV}$, there is a node at $R=0.76$ and $0.9 \mathrm{fm}$ respectively, indicating that it is a radial excitation of the nucleon with perhaps some higher Fock space components, such as $N \pi$, $N \eta$ and $N \pi \pi$ states. A variational calculation with dynamical clover fermions at $m_{\pi}=156 \mathrm{MeV}$ has also observed a node at $R \sim 0.8 \mathrm{fm}$ in the Roper wave function [47] in Landau gauge. We note that each Roper wave function in Fig. 3 does not damp off at $\mathrm{R}=L / 2=1.6 \mathrm{fm}$ in Fig. 3 which indicates that there will be certain finite size effect for the wave function. As far as the spectrum is concerned, however, $m_{\pi} L=10$ for $m_{\pi}=633 \mathrm{MeV}$ (upper panel) and 7 for $m_{\pi}=438 \mathrm{MeV}$ (lower panel). Thus, we expect that the Roper mass is not much affected. The purpose of Fig. 3 is to reveal that the Roper is mainly a radial excitation with a node in its radial wave function. The finite size effect of the wave function is not a primary concern in this study.

The existence of a node in the Roper wave function explains the observation that the spectral weight for the 

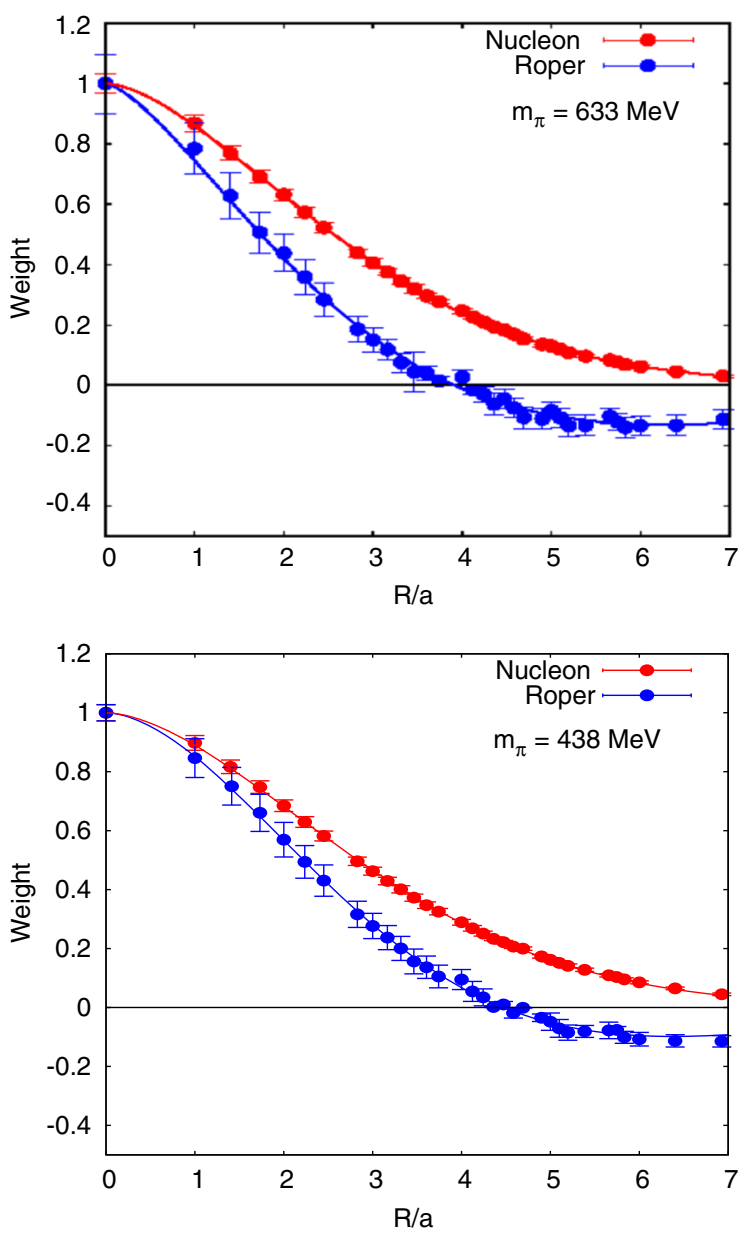

FIG. 3. Nucleon and Roper wave functions in the Coulomb gauge for two different pion masses.

Roper from the Coulomb wall source and point sink is negative, in contrast to that of the nucleon. Since the spectral weight of the Roper with the Coulomb wall source is the product of two factors, one of which is the positive matrix element $\left\langle 0\left|\sum_{x} \chi(x, x, x)\right|\right.$ Roper $\rangle$ for the sink, the sign of the wall-source matrix element $\left\langle\operatorname{Roper}\left|\sum_{x, y, z} \chi(x, y, z)\right| 0\right\rangle$ controls the overall sign. The extra spatial sums in this matrix element involve an $R^{2}$ measure which gives more weight to positions with larger $R$, so the node in the Roper wave function (seen in Fig. 3) turns the overall weight negative. Thus, both the explicit calculation of the radial wave function and the negative spectral weight lend concrete evidence to the fact that the Roper is primarily a radial excitation of the nucleon with perhaps some higher Fock-space components. These lattice results have ruled out that the Roper is a pure pentaquark state, since such a state would have one quark or anti-quark in the relative P-wave and the rest in the lowest $\mathrm{S}$-waves. This would not lead to a radial node in the BetheSalpeter wave function, in contrast to observation.

In view of the fact that the node of the Roper occurs at the radial distance of $\sim 0.9 \mathrm{fm}$ for the light pion $\left(m_{\pi}=438 \mathrm{MeV}\right)$ case, one would want to have a source
TABLE II. Values used for the smearing parameter $w$ for the clover and overlap variational calculations, the number of iterations used for the Gaussian smearing, and the measured root-mean-square (rms) radius.

\begin{tabular}{lccllcc}
\hline \hline \multicolumn{2}{ll}{ Clover } & & & \multicolumn{3}{l}{ Overlap } \\
\cline { 1 - 2 } \cline { 5 - 7 }$w$ & Iterations & $\left\langle r^{2}\right\rangle^{1 / 2}(\mathrm{fm})$ & & Iterations & $\left\langle r^{2}\right\rangle^{1 / 2}(\mathrm{fm})$ \\
\hline 0 & 0 & 0 & & 0 & 0 & 0 \\
2 & 50 & 0.19 & & 50 & 0.16 \\
4 & 100 & 0.39 & & 4 & 100 & 0.34 \\
7 & 200 & 0.63 & & 8 & 100 & 0.63 \\
11 & 400 & 0.86 & & 12 & 800 & 0.85 \\
\hline \hline
\end{tabular}

with a commensurate size so that it could differentiate the nucleon from the Roper in the variational calculation. In this regard, we have chosen 5 operators with different Gaussian smearing sizes whose root-mean-square (rms) radius ranges from 0 to $0.86 \mathrm{fm}$, which should be enough to cover the expected Roper node at $\sim 0.8-0.9 \mathrm{fm}$. Values chosen for the smearing parameter $w[48]$ and the number of iterations, and the corresponding rms radius are listed in Table II. We solve the equation for the generalized eigenvalue problem (GEVP) [49-51]

$$
C(t) v_{n}\left(t, t_{0}\right)=\lambda\left(t, t_{0}\right) C\left(t_{0}\right) v_{n}\left(t, t_{0}\right)
$$

where $C(t)$ is the $N \times N$ correlator matrix for $N$ interpolation field operators $O_{i}$ with matrix element defined as

$$
C_{i j}(t)=\left\langle O_{i}(t) O_{j}^{*}(0)\right\rangle,
$$

and the $n$th eigenvalue is expected to be [52]

$$
\lambda_{n}\left(t, t_{0}\right)=e^{-E_{n}\left(t-t_{0}\right)}\left(1+\mathcal{O}\left(e^{-|\delta E|\left(t-t_{0}\right)}\right),\right.
$$

where $\delta E$ is the energy gap between $E_{n+1}$ and $E_{n}$. We have used 761 configurations of the isotropic clover action, on each of which we chose 36 time slices to place the source, resulting in 27,396 measurements in total. Spatially, the sources are randomly placed. With the smearing parameters $w=0,4,7$ and 11 , we find the first nucleon excited state at 1.92(6) GeV as shown in Fig. 4(a) for the case $t_{0}=2$. This (red point in Fig. 2) is quite consistent with that from SEB method at 1.87(13) GeV (blue point in Fig. 2). On the other hand, when a set of interpolation fields with only small smearing sizes ( $w=0,2$ and 4$)$ are used, we find the first excited state at 2.19(11) GeV as shown in Fig. 4(b) (black point in Fig. 2), which agrees with the JLab results.

This suggests that there are two radially excited states of the nucleon. The lower one is mainly the $2 \mathrm{~S}$ state with a radial node at $0.8-0.9 \mathrm{fm}$ as illustrated in Fig. 3, while the higher one could be the $3 \mathrm{~S}$ state with the first radial node smaller than that of the $2 \mathrm{~S}$ as evidenced in the wave function study of Ref. [53], which is characteristic of the 


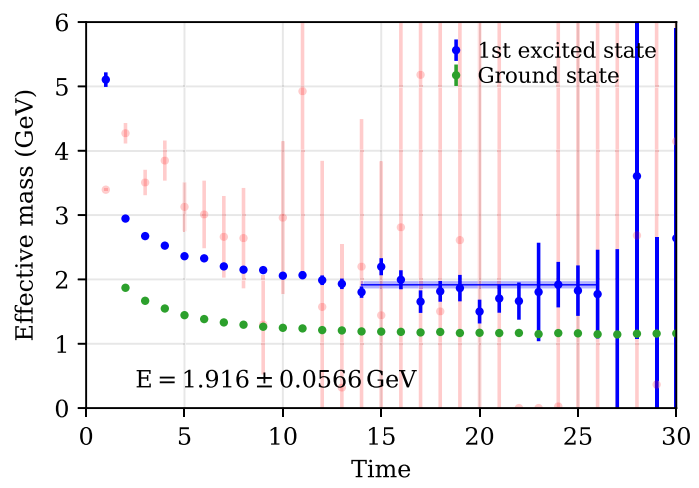

(a) Result from smear parameter $w=0,4,7$, and 11 .

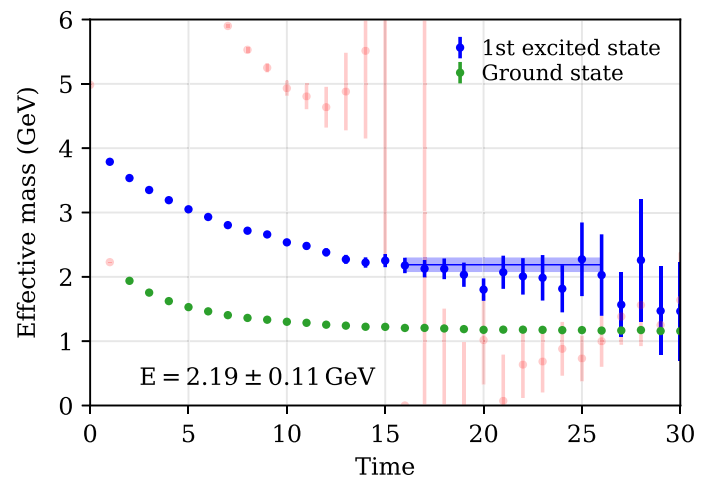

(b) Result from smear parameters $w=0,2$, and 4 .

FIG. 4. Effective mass of the states extracted from the variation study. Some states are painted faintly for clarity. The blue horizontal line and band shows the fit of Roper mass.

Airy function as the solution of a linear potential. As a result, it has better overlap onto the interpolating source and sink with a smaller size. The second excited state may well be the $N(1710) 1 / 2^{+}$at $1700 \mathrm{MeV}$ which is $\sim 330 \mathrm{MeV}$ above the Roper, which is about the same gap we see between the two excited states in the clover case. Since the variational method with large smeared operators agrees with the SEB method, it shows that both approaches are feasible in obtaining the first excited state, namely the Roper.

\section{A. Variational calculation with the overlap fermion}

Next, we move on to performing variational calculations for the DWF configurations with valence overlap fermions to check the validity of the results from the SEB method as alluded to earlier. We shall first consider the set of 4 smearing sizes in Table II with $w=0,2,4$ and 8. Before solving Eq. (2), we first determine the combination of interpolation operators which can lead to well-separated nondegenerate states as is carried out in Ref. [33]. To do so, we solve for the eigenvalues and eigenvectors of the $4 \times 4$ correlation matrix $C(t)$ in Eq. (3)

$$
C(t) u_{n}(t)=E_{n}(t) u_{n}(t)
$$

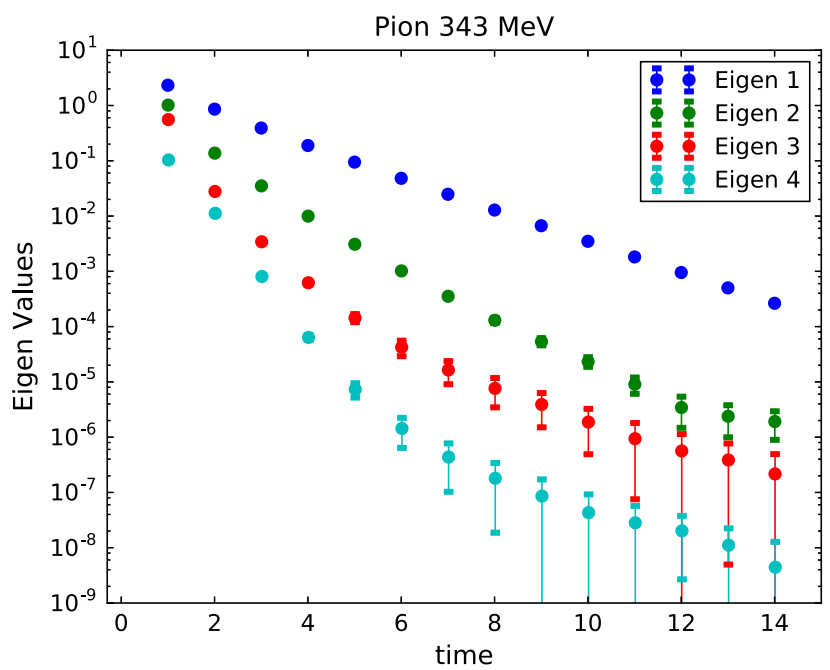

(a) Eigenvalues of the $4 \times 4$ correlation matrix as a function of $t$ for for $m_{\pi}=343 \mathrm{MeV}$.

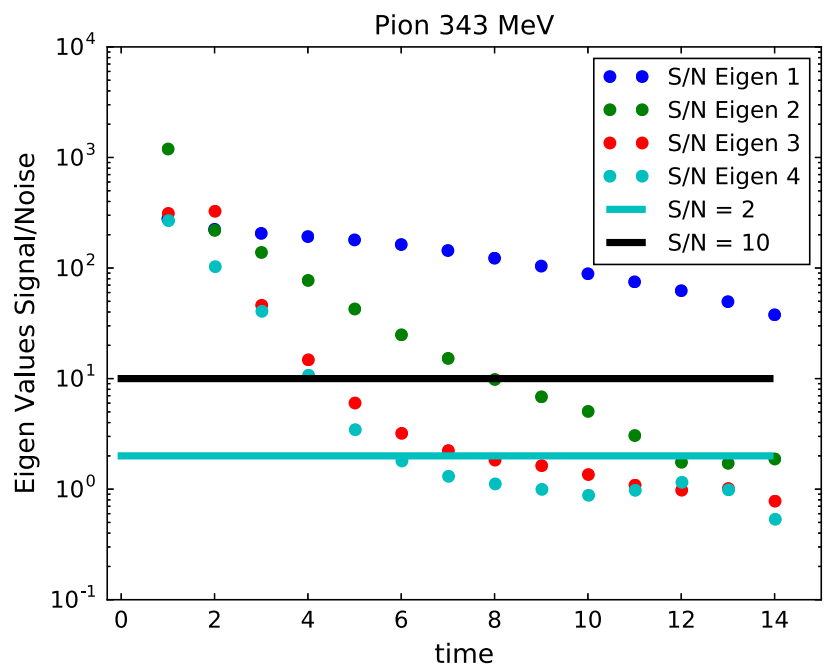

(b) The signal-to-noise ratio of the 4 eigenvalues as a function of $t$ for $m_{\pi}=343 \mathrm{MeV}$. Also plotted are the $\mathrm{S} / \mathrm{N}=2$ and 10 lines.

FIG. 5. Eigenvalues and the signal-to-noise ratio $\mathrm{S} / \mathrm{N}$ as a function of time for $m_{\pi}=343 \mathrm{MeV}$.

Note that $E_{n}(t)$ here is not the same as $\lambda\left(t, t_{0}\right)$ in Eg. (2). We plot the eigenvalues of $C(t)$ in Fig. 5(a) and their signalto-noise $(\mathrm{S} / \mathrm{N})$ ratios in Fig. 5(b) as a function of $t$ for the case $m_{\pi}=343 \mathrm{MeV}$. We see from Fig. 5(b) that the S/N ratios of the two lower eigenvalues fall below $\mathrm{S} / \mathrm{N}=2$ beyond $t=8$. This is where the first excited state starts to level off. Thus, we are only able to resolve two states from this set of smeared operators with the projected matrix

$$
\tilde{C}(t)=U^{T} C(t) U
$$

where $U=\left[u_{1}, u_{2}\right]$ is the $4 \times 2$ matrix spanned by the first two eigenvectors with acceptable $\mathrm{S} / \mathrm{N}$ ratios (i.e., $\geq 10$ ), for 


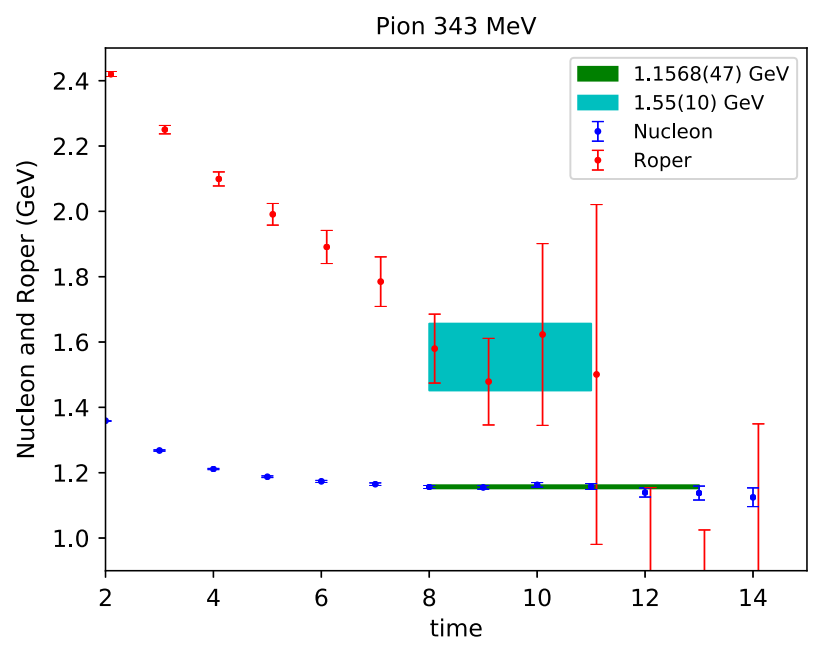

(a) Nucleon and Roper effective masses for $m_{\pi}=343 \mathrm{MeV}$ as a function of $t$. The error bands are from the single exponential fits.

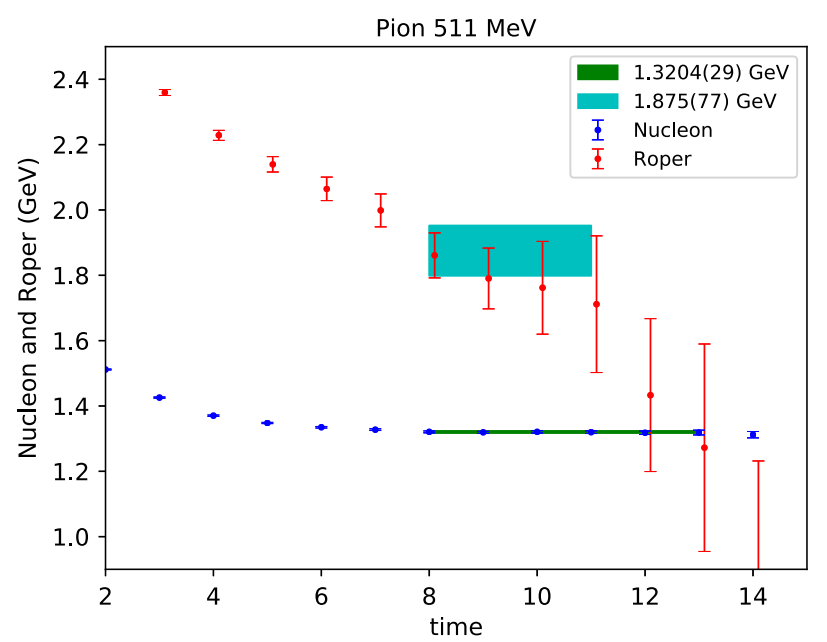

(b) The same as in Fig. 6 a for $m_{\pi}=511 \mathrm{MeV}$.

FIG. 6. Results of the variational calculations of overlap fermion on $2+1$ fermion DWF configurations for two valence pion masses. These are from the eigenvector-projected variation with $w=0,2,4$ and 8 smearing.

their eigenvalues at $t=8$. We then solve the projected GEVP for the projected $2 \times 2 \tilde{C}(t)$

$$
\tilde{C}(t) v_{n}\left(t, t_{0}\right)=\lambda\left(t, t_{0}\right) \tilde{C}\left(t_{0}\right) v_{n}\left(t, t_{0}\right),
$$

at the projection time $t_{\text {pro }}=8$ and initial $t_{0}=2$. The effective mass is defined as

$M_{n}(t)=\ln \left[\left(v_{n}^{T}(t-1) \tilde{C}(t-1) v_{n}(t-1)\right) /\left(v_{n}^{T}(t) \tilde{C}(t) v_{n}(t)\right)\right]$

where $M_{1}$ is the nucleon mass and $M_{2}$ is that of the first excited state and they are plotted in Fig. 6(a) for $m_{\pi}=$ $343 \mathrm{MeV}$ and in Fig. 6(b) for the partially quenched valence $m_{\pi}=511 \mathrm{MeV}$. The bands in these figures show the errors of the fit of $\left(v_{n}^{T}(t) \tilde{C}(t) v_{n}(t)\right)$ with a single exponential $e^{-M_{n}\left(t-t_{0}\right)}$ in the chosen windows which start at $t=8$. It is gratifying to see that the Roper mass $M_{R}=$ $1.55(10) \mathrm{GeV}$ at $m_{\pi}=343 \mathrm{MeV}$ is in good agreement with the corresponding value of $1.60(12) \mathrm{GeV}$ from the SEB method.

To check the sensitivity of the excited state to the smearing size and the related issue of a possible second excited state, as alluded to in the study of the anisotropic clover lattice discussed in Sec. III, we employ additional variational calculations with different smearing sizes for the nucleon source. We consider a combination of a Coulomb wall and a Gaussian smeared source and gauge-invariant box smeared sinks [54] in the variational calculation. The gauge-invariant box smearing is more economical than Gaussian smearing, especially when the smearing size is large [54]. We shall use multiple box sizes for the sink. This entails variational calculations with asymmetric correlation matrices. Variational approach with asymmetric correlation matrices has been applied to the excitation spectrum of baryons, particularly the positive- and negative-parity excited states of the nucleon $[21,55,56]$. The size of the smeared box $B_{N}$ is $N$ lattice spacings and the physical length is $L_{B_{N}}=N a$, where $a=0.1105 \mathrm{fm}$ for this lattice. Besides the wall source, we have another Gaussian smeared source with $w=5.5,8$ or 12 . The rms radii for the sources with $w=8$ and 12 are $0.63 \mathrm{fm}$ and $0.85 \mathrm{fm}$, respectively, as listed in Table II, and that of $w=5.5$ is $0.46 \mathrm{fm}$. In each case, we used two box sources $\left(B_{N}\right)$ to solve the generalized eigenvalue problem (GEVP) for the $2 \times 2$ asymmetric correlation matrix for each $t>t_{0}$. The specifics of the source and sink, and $t_{0}, t_{\text {ref }}$ are given for a total of 9 different setups in Table III. The eigenvalues $e^{-m_{i} t}$ and eigenvectors are real for the asymmetric correlation matrix so defined. The effective mass and $\left(v_{n}^{T}(t) \tilde{C}(t) v_{n}(t)\right)$ are defined from the eigenvectors at $t_{\text {ref }}$ as specified in Table III. It turns out that all the methods with different

TABLE III. Test of sensitivity to the size of the Gaussian source. A Coulomb and a Gaussian source with three sizes are tested. Gauge-invariant box sinks $B_{N}(\mathrm{~N}=2,4,6,8,12)$ are listed for each of the three combined Coulomb and smeared sources.

\begin{tabular}{lcccc}
\hline \hline Method & Source & Sink & $t_{0}$ & $t_{\text {ref }}$ \\
\hline 1 & Wall, $\omega 12$ & B2, B6 & 5 & 6 \\
2 & Wall, $\omega 12$ & B4, B8 & 5 & 6 \\
3 & Wall, $\omega 12$ & B8, B12 & 4 & 6 \\
4 & Wall, $\omega 8$ & B2, B6 & 4 & 6 \\
5 & Wall, $\omega 8$ & B4, B8 & 4 & 6 \\
6 & Wall, $\omega 8$ & B8, B12 & 4 & 6 \\
7 & Wall, $\omega 5.5$ & B2, B6 & 3 & 6 \\
8 & Wall, $\omega 5.5$ & B4, B8 & 3 & 6 \\
9 & Wall, $\omega 5.5$ & B8, B12 & 3 & 6 \\
\hline \hline
\end{tabular}




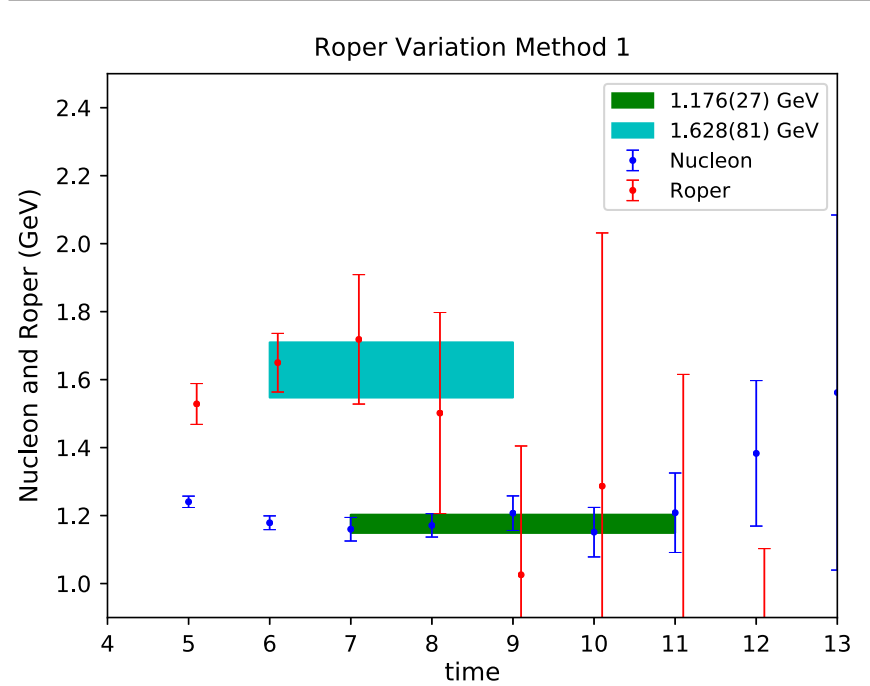

(a) Nucleon and Roper effective mass for method 1 in Table III.

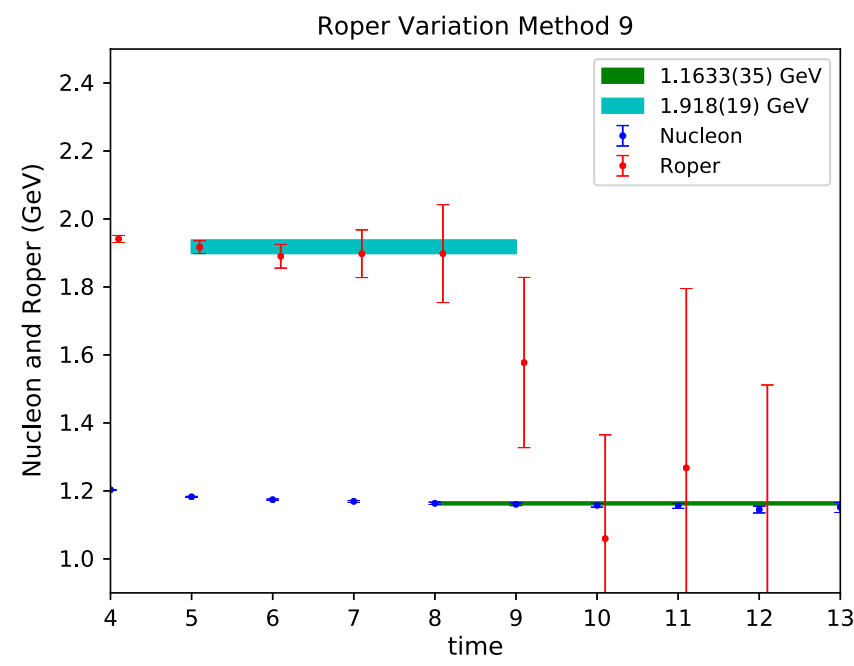

(b) Same as in Fig. 7a for method 9.

FIG. 7. Results from variational calculation of overlap fermion on $2+1$ fermion DWF configurations at $m_{\pi}=343 \mathrm{MeV}$ with different sources and sinks whose specifics are given in Table III.

source sizes produce the same nucleon mass for $m_{\pi}=$ $343 \mathrm{MeV}$, but a range of Roper masses from 1.63(8) GeV for method 1 to $1.92(2) \mathrm{GeV}$ for method 9. The effective masses for the nucleon and Roper for methods 1 and 9 are plotted in Fig. 7, similar to those in Fig. 6. We present the nucleon and the Roper results in Fig. 8 for the 9 methods in Table III together with that with 4 sources and 4 sinks from Fig. 6(a). We see that, again, the sources with large smearing ( $w=12$ for method 1, 2 and 3) (colored green) have lower masses than those with smaller smearing (red points with $w=8$ ) and still smaller smearing (cyan points with $w=5.5$ ). This is basically the same as we found with the anisotropic clover lattice in Sec. III, i.e., the use of the smaller smearing sources results in a higher value for the measured excited-state mass. This is consistent with the

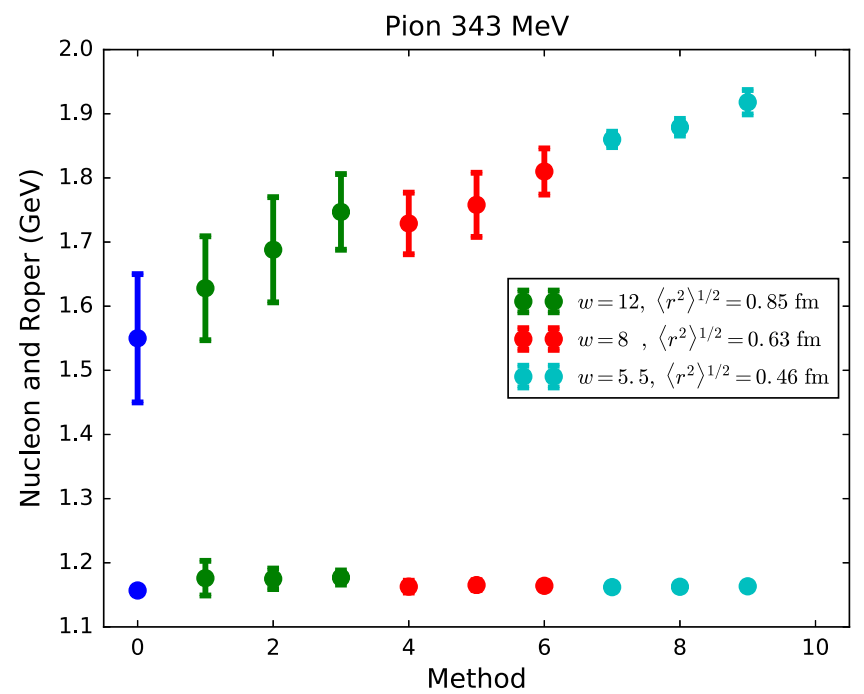

FIG. 8. The nucleon and first excited state in the $1 / 2^{+}$channel for 9 methods with various sources and sinks listed in Table III. Method 0 is the case with eigenvector-projected variation with $w=0,2,4$ and 8 smearing. These are for the case $m_{\pi}=343 \mathrm{MeV}$.

picture that there are two radially excited states, namely the Roper and $N(1710) 1 / 2^{+}$at $1700 \mathrm{MeV}$, which is $\sim 330 \mathrm{MeV}$ above the Roper. The latter, being 3S radial excitation with a smaller node position overlaps better with a source of a smaller size. In Fig. 8, an almost monotonic increase of the measured value of the excited-state mass as the source size decreases. This suggests that the small $2 \times 2$ correlation matrix results in a contamination between the first and second excited states in the windows amenable to fits with reasonable errors. The clear signal in Fig. 7(b) suggests that it is the pure second excited state. With the present setup and statistics, we are not able to resolve 3 separated states with the chosen set of operators. Otherwise, it would have been easier to verify the picture that we infer.

We add the results of the variational calculation with $w=0,2,4$ or 8 on Fig. 2 (brown points) for several pion masses to show that they are consistent with those from the SEB method. The upshot of the variational calculation with large smearing size is that its results are consistent with those from the SEB method for both the clover and overlap fermions. This removes the uncertainty regarding the fitting algorithms. The remaining challenge is to understand why the Roper with overlap fermion is lower than that from the clover fermion by $\sim 300 \mathrm{MeV}$ on lattices with similar lattice spacing and sea quark mass.

We have not studied the source-size dependence of SEB, but we presume the size will have an effect, similar to what is found in the variational approach. In this work, we used the Coulomb wall source for the overlap data on the $24^{3} \times$ 64 lattice in Sec. II which are shown in Fig. 2 as the pink points. The wall source is known to suppress the higher excited states so that the first excited state can be extracted 
more readily in the fitting algorithm, such as SEB. Had we used a point source, the SEB would have given a high first excited state, similar to that of the variational approach with small smearing sources. This is so because all the $\mathrm{nS}$ radially excited states would have contributed coherently to the spectral weights for the point source and the present lattice spacing at $0.114 \mathrm{fm}$ is not fine enough to isolate the $2 \mathrm{~S}$ state from the $3 \mathrm{~S}$ and higher excited states. It is conceivable that varying the size of the source in SEB may well result in a mixture of $2 \mathrm{~S}$ and $3 \mathrm{~S}$ states as shown in the variational approach in Fig. 8. We will study this in the future.

We should mention that the Roper is a resonance which decays to $N \pi, N \pi \pi, \Delta \pi \cdots \ldots$ To find its pole mass and width on the lattice, one should do the scattering phase shift calculation including the $N \pi$ channel with the Luscher method, such as those that have been done in Refs. [37,38]. The Luscher method relates the interaction energy shift of the two hadrons to the phase shift. In the case of the Roper, this energy shift is a reflection of the mixing between the confined would-be Roper from the 3-quark interpolation field and the $\pi N$ P-wave scattering state at a particular relative momentum. As the two states approach each other when the relative momentum between the $\pi$ and $N$ changes, there is level repulsion to avoid crossing. The maximum repulsion occurs at maximum mixing of the two states and they are positioned at the half maxima of the scattering cross section (or phase shift $\delta=90$ degrees) sandwiching the maximum point for a Breit-Wigner resonance cross section, which reflects the full-width-at-half-maximum (FWHM). When the width of the resonance is relatively narrow (e.g., the Roper width is $\sim 175 \mathrm{MeV}$ ) compared to its mass, the maximum repulsion occurs close to the wouldbe crossing point between the noninteracting two hadron state (i.e., $\pi N$ ) and the confined would-be Roper from the 3 -quark interpolation field. When the lattice volume is large enough so that the would-be Roper does not change appreciably with increasing volume, the would-be crossing point is just the mass of the would-be Roper. In this case, the Roper mass from the 3-quark interpolation field in this work should be close to the resonance pole calculated from the $\pi N$ scattering study à la Luscher. The shift of the pole position from the would-be Roper mass is related to the deviation from the Breit-Wigner form and is bounded by half of the width $\Gamma / 2$ which is $\sim 88 \mathrm{MeV}$ for the Roper. This is much smaller than the $\sim 600 \mathrm{MeV}$ discrepancy between the Roper states calculated from the clover and overlap fermions, which is what we try to resolve in this study. We can find evidence for the above argument from the lattice calculations of the $\rho$ resonance from $\pi \pi$ scattering. One is a $N_{f}=2$ calculation of the $\rho$ resonance from $\pi \pi$ scattering in the elongated lattice to change the pion momentum [57]. The resonance mass $a m_{\text {res }}=0.4878(4)$ turns out to be very close to the would-be $\rho$ mass of $a m_{\rho}=$ $0.4800(30)$ [58] from the $\bar{q} \gamma_{i} q$ interpolation field at a volume where the $\pi \pi \mathrm{P}$-wave state is far above the wouldbe $\rho$. The other $\pi \pi+K \bar{K}$ calculation [59] is carried out on an isotropic lattice for the $N_{f}=2+1$ case; the resonance mass is again very close to that from the $\bar{q} \Gamma q$ interpolators. Since the width of the Roper at $175 \mathrm{MeV}$ is close to that of the $\rho$ at $150 \mathrm{MeV}$, we think that using 3-quark interpolation field to calculate the Roper mass in this work should be a good approximation to that of the Roper resonance.

\section{MULTIHADRON STATE FROM SINGLE- HADRON INTERPOLATION FIELD}

To track down the origin of the difference in the prediction of the Roper mass in clover and overlap fermions, we note that chiral effective theory hints toward substantial mixtures of higher Fock-space components, involving $N \pi$, and $N \pi \pi$ states, as the reason why the quark model's Roper prediction is too high. As mentioned in the introduction in Sec. I, the most sophisticated dynamical coupled-channel (DCC) model [9-11] shows that the coupling to $\pi N, \eta N$ and $\pi \pi N$ brings down the uncoupled bare Roper state by $\sim 400 \mathrm{MeV}$ to the experimental value. The experimental electroexcitation amplitudes of the Roper resonance provides evidence for it to be primarily the radial excitation of a three-quark core, while higher Fock space components are needed to describe the low $Q^{2}$ behavior of the amplitudes [60].

To study the implication of the higher Fock space and the difference between the clover and overlap fermions, we note that there is a well-documented would-be $\eta \pi$ ghost state in the isovector scalar meson channel (i.e., the $a_{0}$ channel) in the quenched approximation with overlap fermion [41]. This is caused by the "hairpin" diagram as illustrated in Fig. 9 with the $\bar{\psi} \gamma$ interpolation field.

It is clearly observed that the $a_{0}$ correlator starts to develop a negative tail when the pion mass is less than $\sim 600 \mathrm{MeV}$, and it is progressively more negative at earlier time slices for smaller quark masses. This is a clear indication that at least one of the ghost $\eta \pi$ states, being lightest in mass, is dominating the correlator over the physical $a_{0}$ at larger times. This has been reported in the literature for the quenched [61-63] and partially quenched [64] calculations. This ghost $\eta \pi$ contribution in the $a_{0}$ correlator has been studied in the chiral perturbation theory $[41,61,64]$. The Fourier transform of the one-loop hairpin

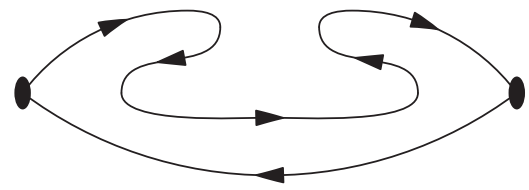

FIG. 9. Hairpin diagram in the quenched isovector scalar meson $\left(a_{0}\right)$ correlator. When the pion mass is lower than $\sim 600 \mathrm{MeV}$, it produces a ghost would-be- $\eta \pi$ state which shows up as a negative tail at large time separation for the overlap fermion. 


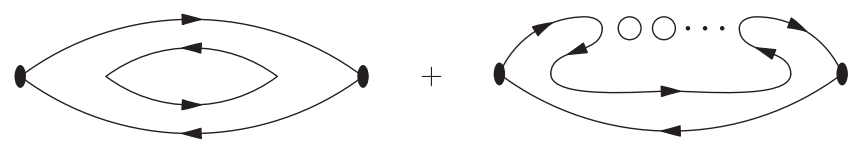

FIG. 10. The isovector scalar meson $\left(a_{0}\right)$ correlator with dynamical fermion configurations. The quark loops in the vacuum are responsible for producing full $\eta \pi$ and $\eta^{\prime} \pi$ propagators in the $2+1$-flavor case.

diagram gives the following contribution to the isovector scalar meson propagator in Euclidean time [41]

$$
\begin{aligned}
G_{S}(\vec{p}=0) & =F T\left\{a^{2} \Delta_{h}(\vec{p}=0)\right\} \\
& =-\frac{r_{0}^{2} m_{0}^{2}}{2 N_{S}^{3}} \frac{\left(1+m_{\pi} t\right)}{2 m_{\pi}^{4}} e^{-2 m_{\pi} t}+\left(t \rightarrow N_{T}-t\right),
\end{aligned}
$$

where the $\left(1+m_{\pi} t\right)$ factor is due to the double pole of the would-be $\eta$ ghost propagator in the loop. $r_{0}$ is the coupling between the scalar interpolation field and the $\eta$ and $\pi$ or the matrix element $\langle 0|\bar{\psi} \psi| \eta \pi\rangle$ and $m_{0}^{2}=2 N_{f} \chi_{t} / f_{\pi}^{2}$ is the hairpin insertion mass which is related to the topological susceptibility $\chi_{t}$ in the pure gauge theory. Note this ghost state contribution is negative due to the coupling between two annihilating pseudoscalar propagators in the unfinished $\eta$ channel without the presence of quark loops as in Fig. 10.

In a full dynamical fermion situation, the quark loops from the fermion determinant give rise to a geometrical series to lift the would-be Goldstone boson to $\eta$ and $\eta^{\prime}$ in the Witten-Veneziano formalism to resolve the $U(1)$ anomaly [65,66]. This is illustrated in the cartoon picture in Fig. 10. In the quenched approximation, there are no quark loops. This results in the hairpin diagram from the double annihilation of the would-be $\eta$ in Fig. 9 and gives the negative ghost $\eta \pi$ behavior in the quenched $a_{0}$ correlator $[41,61,64]$. The existence of this ghost propagator is a prelude to revealing the fact that the $\eta \pi$ type multihadron states can be produced by the one-hadron $\bar{q} q$ interpolation field in a full dynamical fermion calculation. Even though this ghost $\eta \pi$ behavior is well documented in a quenched calculation with the overlap fermion for a range of pion mass from $600 \mathrm{MeV}$ down to $180 \mathrm{MeV}$, there has not been such clear evidence for the Wilson-type fermion. We shall make a comparison between the overlap fermion and the clover fermion.

Plotted in Fig. 11 are the $a_{0}$ correlators on three quenched Wilson-gauge lattices with lattice size/spacing of $24^{3} \times 48 / 0.12 \mathrm{fm}$ (upper panel), $28^{3} \times 48 / 0.09 \mathrm{fm}$ (middle panel), and $32^{3} \times 64 / 0.06 \mathrm{fm}$ (lower panel), respectively. The $a_{0}$ correlators are calculated at $\sim 296 \mathrm{MeV}$ pion mass for the overlap and clover fermions with the mean-field clover term. Both of them are HYPsmeared. To take into account the fact that the renormalization factors $Z_{S}$ are different in the two actions, we compare their $a_{0}$ correlators for the heavy pion case
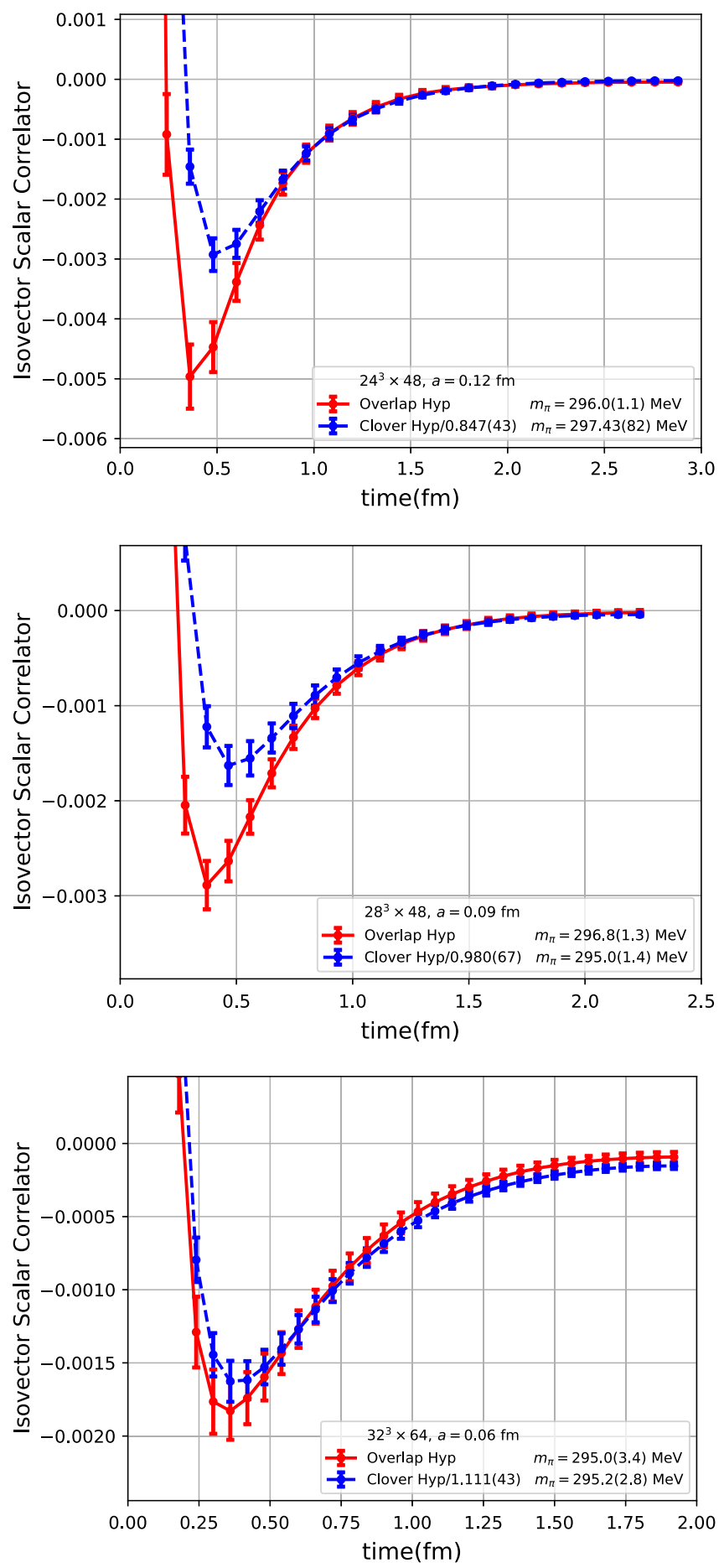

FIG. 11. Comparing the isovector scalar meson $\left(a_{0}\right)$ correlators between clover and overlap fermions for pion masses at $\sim 296 \mathrm{MeV}$ for lattice spacings $a=0.12 \mathrm{fm}$ (upper panel), $a=$ $0.09 \mathrm{fm}$ (middle panel) and $a=0.06 \mathrm{fm}$ (lower panel). The lines connecting the points are there to guide the eyes.

$(\sim 1 \mathrm{GeV})$ where there are no ghost states. To normalize the correlators for comparison in Fig. 13, we divide the clover $a_{0}$ correlator by a factor which is the ratio of the clover to overlap correlator values at large time separation 
with the $a_{0}$ mass at $\sim 1.6-1.9 \mathrm{GeV}$. The factors are $0.847(43), 0.980(67), 1.111(43)$ for the lattices with spacings $0.12,0.09$ and $0.06 \mathrm{fm}$, respectively.

The $\eta \pi$ ghost states are clearly seen in all three lattices where the correlators turn negative beyond $t \sim 0.25 \mathrm{fm}$. The conspicuous feature of the comparison is that the negative parts of the clover correlators for the cases of $a=0.12 \mathrm{fm}$ (upper panel) and $a=0.09 \mathrm{fm}$ (middle panel) are shallower than those of the overlap, whereas they are about the same at $a=0.06 \mathrm{fm}$ (lower panel). The existence of the minimum is the result of a positive exponential term from the physical $a_{0}(1236)$ and a negative exponential term from the $\eta \pi$ ghost in Eq. (9). Since we have taken the relative renormalization of the clover and the overlap into account from comparison of the $a_{0}$ correlator at large pion mass where there is no ghost state, the physical $a_{0}$ exponential should be the same for the cases in Fig. 11. Their differences should reflect the couplings to the $\eta \pi$ ghost state. Therefore, we take the correlator at the minimum to serve as an approximate yet meaningful indicator for the strength of coupling to the $\eta \pi$ state. To see the quark mass dependence, we calculate the ratio of the $a_{0}$ correlator between the clover and overlap fermions at their respective minimum in the pion mass range between $250 \mathrm{MeV}$ and $450 \mathrm{MeV}$ and plot in Fig. 12. We see that for $a=0.12 \mathrm{fm}$ and $0.09 \mathrm{fm}$ the ratios are about $0.4-0.6$ and the pion mass dependence in this range is not strong. On the other hand, the ratio is close to unity for the $a=0.06 \mathrm{fm}$ case across the same mass range. This basically reflects what we have seen in Fig. 11. It is commonly believed that chiral symmetry is recovered for the Wilson-type fermion in the continuum limit; we interpret our results to imply that the chiral symmetry breaking effect of the clover fermion is

Ratio of Clover/Overlap Minmum

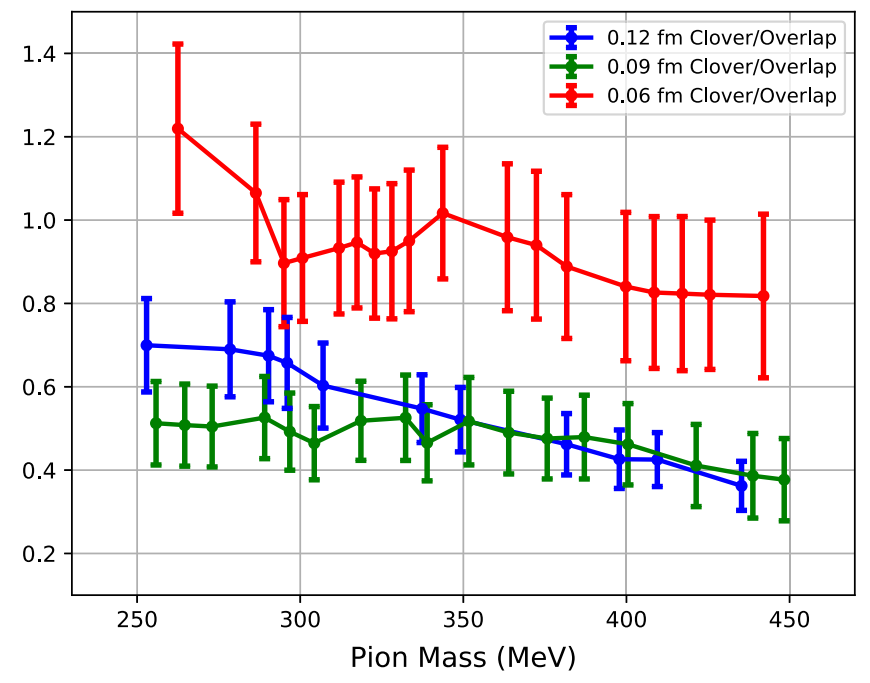

FIG. 12. The ratios of the minima of the quenched $a_{0}$ correlator of the clover fermion to that of overlap fermion for a range of pion masses in three lattice spacings. indeed observable at $a=0.12 \mathrm{fm}$ and $a=0.09 \mathrm{fm}$, whereas the chiral symmetry is likely recovered for the clover at $a=0.06 \mathrm{fm}$, by virtue of the fact that its $a_{0}$ correlator, including the coupling to the $\eta \pi$ ghost state, seems to coincide with that of the overlap fermion. These can be considered discretization error. However, it is not clear if what we observe is a simple $\mathcal{O}\left(a^{2}\right)$ error. In view of the fact that the ratios are at $\sim 0.5$ for both the $a=0.12 \mathrm{fm}$ and $a=0.09 \mathrm{fm}$ cases and they jump to unity at $a=0.06 \mathrm{fm}$, the possibility that chiral symmetry restoration may set in abruptly near the latter lattice spacing should be considered.

We have also compared Wilson and overlap fermions to explore the discretization errors related to chiral dynamics. Plotted in Fig. 13 are the $a_{0}$ correlators from the Wilson and overlap fermions (without HYP smearing) on the same three quenched Wilson-gauge lattices with a pion mass around $290 \mathrm{MeV}$. As in the case of the above clover/overlap comparison, we divide the Wilson $a_{0}$ correlator by a factor which is the ratio of the Wilson to overlap correlator values at large time separation with the $a_{0}$ mass at $\sim 1.7 \mathrm{GeV}$. The factors are 2.43(52), 2.20(20), 2.20(16) for the lattices with spacings $0.12,0.09$ and $0.06 \mathrm{fm}$, respectively. To put results from all three lattices in the same figure, we have multiplied the $N_{S}^{3}$ factor from Eq. (9) to scale out the volume dependence as far as the ghost state is concerned. We see from Fig. 13 that the ghost states in the Wilson fermions are much shallower than those the overlap. They are shallower than those of the clover fermion in Fig. 11. When the ratios of the minima are plotted in Fig. 14 for the pion mass range of 280 to $330 \mathrm{MeV}$, we see that the Wilson minima is only $\sim 10 \%$ of that of the overlap for the $a=$ 0.12 and $0.09 \mathrm{fm}$ cases. At $a=0.06 \mathrm{frm}$, the ratio is still only $\sim 40 \%$. This clearly demonstrates that Wilson fermion

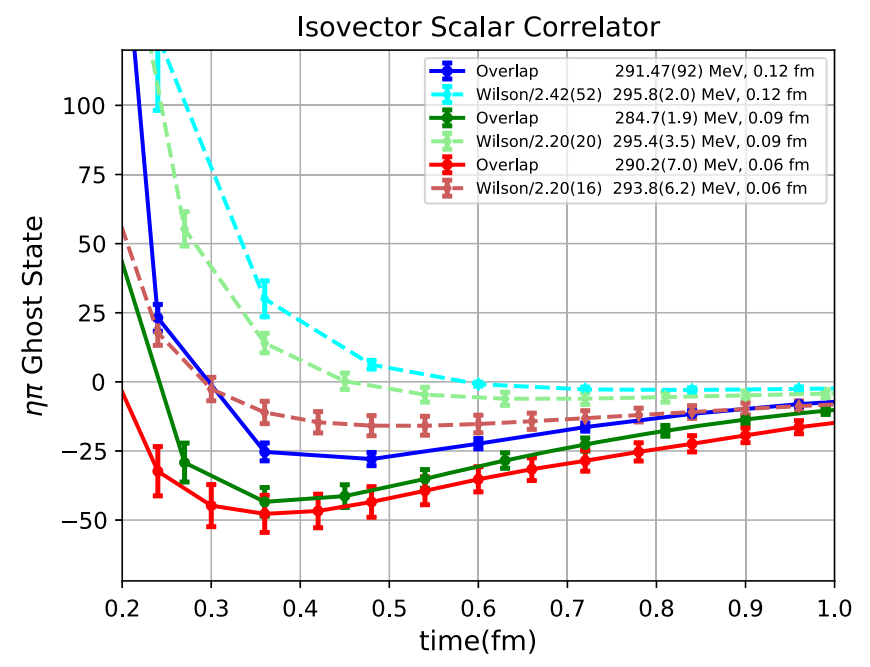

FIG. 13. The isovector scalar meson $\left(a_{0}\right)$ correlator with quenched configurations for Wilson and overlap fermions for a valence pion mass of $\sim 290 \mathrm{MeV}$ at three lattice spacings are plotted for comparison. 


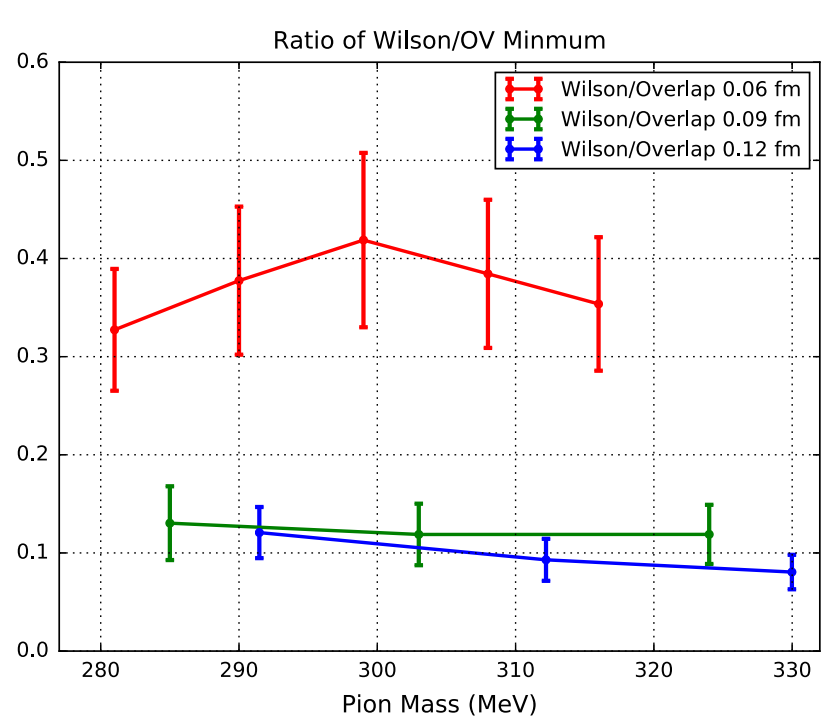

FIG. 14. The ratios of the minima of the quenched $a_{0}$ correlator of the Wilson fermion to that of overlap fermion for a range of pion masses at three lattice spacings.

at these lattice spacings, due to a lack of chiral symmetry, does not produce the full $Z$-graphs in the hairpin diagram, as does the overlap fermion. Its coupling to the $\eta \pi$ ghost is still weaker than that of the clover. This is to be expected since Wilson has $\mathcal{O}(a)$ error, whereas clover has $\mathcal{O}\left(a^{2}\right)$ error and is chirally improved from the Wilson fermion.

How is the above observation of the ghost state in the $a_{0}$ channel related to the fact that the Roper state is $\sim 300 \mathrm{MeV}$ lower than that of the clover fermion that we studied in Sec. III? As we mentioned in the Introduction: the finding in the extensive dynamical coupled-channel (DCC) study [9-11], the need of higher Fock space in the electroexcitation experiment [60], and the pattern of parity reversal of the excited $N, \Delta$ in contrast to those of $\Lambda$, all lead to the suggestion that there is a large higher Fock space component in the Roper state. It is shown explicitly in a chiral constituent quark model that the flavor-spin interaction, due to the pseudoscalar meson exchange between the quarks, is responsible for the parity pattern in $N, \Delta$, and $\Lambda$ and the lowering of Roper from the $S U(6)$ quark model with the color-spin interaction [67].

For the baryons, there are quark loop diagrams leading to $\pi N$ and $\eta N$ states which are depicted in Fig. 15. They are analogous to the $\eta \pi$ state in the $a_{0}$ correlator in Fig. 10.

In order to study the impact of the higher Fock-space components on the Roper, lattice calculations with clover fermions at $m_{\pi}=156 \mathrm{MeV}$ are carried out with the inclusion of the $N \pi$ and $N \sigma$ type 5-quark (i.e., $q q q q \bar{q}$ ) interpolation fields in addition to the 3-quark interpolation field. Even though the expected $N \pi \pi$ and $N \pi$ scattering states are observed, no additional state is found below $1.65 \mathrm{GeV}[37,68]$. It is concluded that the Roper does not come down from the value calculated with the 3-quark interpolation field alone in this $\pi N$ scattering calculation.

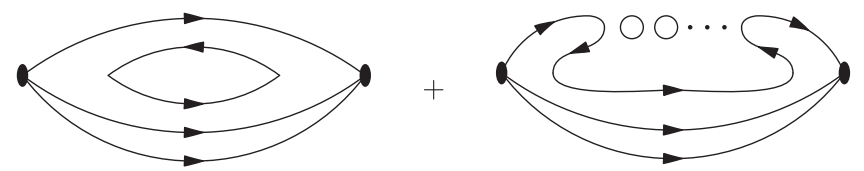

FIG. 15. Quark loop contribution to the $\pi N$ and $\eta N$ intermediate states from a three-quark nucleon interpolation field.

A similar conclusion is reached with mixed 3-quark and 5-quark interpolation fields for the clover fermion at $m_{\pi}=411 \mathrm{MeV}$ [69].

To understand the results of these $\pi N$ scattering calculations, we point out that there are other classes of diagrams that will lead to higher Fock space components. These are depicted in Fig. 16 as Z-graphs. This can happen when there is more than one valence quark (or more than one valence antiquark) in the interpolation field, such as for baryons. The back-bending of the valence quark in the Z-graph results in an antiquark propagating forward in time. It can combine with another forward propagating valence quark to form a meson propagating between the two valence quarks as depicted in Fig. 16. It is worthwhile to note that this mechanism does not exist for the nonsinglet mesons with $\bar{q} \Gamma q$-type interpolation fields [70]. This is because the forward propagating antiquark from the Z-graph due to the valence quark and the other forward propagating antiquark from the interpolation field do not form a meson. To do so, it would require one quark and one antiquark. Thus additional $Z$-graphs in the baryon can be cast effectively as the origin of meson exchanges between the valence quarks in the chiral quark model [71]. It is the flavor-spin interaction from the Goldstone boson exchange that produces the parity-reversal pattern in $N$ and $\Delta$ and lowers the Roper state to its experimental value in the chiral constituent quark model [67]. Including a 5-quark interpolation field will entail coupling to higher Fock-space components, as illustrated in Fig. 15, but not through the $Z$-graphs in Fig. 16, if the fermion action does not have a tendency to generate $Z$-graphs dynamically at the given lattice spacing. The lattice calculations in this study, the $\pi N$ scattering calculations $[37,68,69]$, the Roper calculation in DCC [9-11], and the chiral quark model [67], all together hint strongly that it is the Z-graphs in Fig. 16, innate to chiral dynamics, that are responsible for lowering the Roper state and resulting in the parity reversal in $N$ and $\Delta$ excited states. Therefore, only a lattice action with a good chiral symmetry on the lattice will be able to capture the correct

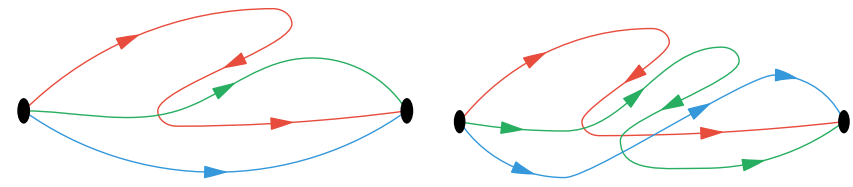

FIG. 16. Z-graphs which lead to $\pi N$ and $\pi \pi N$ intermediate states from a three-quark nucleon interpolation field. 
dynamics of the Roper resonance and aid in the correct extraction of its energy level. Using the overlap fermion action, which has exact chiral symmetry at finite lattice spacing, we are able to extract the energy of the Roper resonance on fairly coarse lattices. On the other hand, at $a=0.06 \mathrm{fm}$ or smaller lattice spacing, the clover fermion may become chiral, in which case the calculated Roper state should come down to its experimental value.

\section{SUMMARY AND CONCLUSION}

We have calculated the Roper state on the lattice from the valence overlap fermions with both the sequential empirical Bayes (SEB) method and the variational method with large smeared sources and found consistent results. The calculations were carried out on the $2+1$-flavor domain-wall fermion gauge configurations on a $24^{3} \times 64$ lattice with the lattice spacing $a=0.114 \mathrm{fm}$ and light sea quark mass which corresponds to a pion mass of $330 \mathrm{MeV}$. The chirally extrapolated Roper mass is consistent with the experimental result of $\mathrm{N}(1440)$.

The Roper masses for pion masses in the range 260$570 \mathrm{MeV}$ are consistently lower than those from the calculation with Wilson-type fermions by $~ 300-600 \mathrm{MeV}$. To further check the fitting algorithms, we applied both SEB and variational approaches to the $2+1$-flavor clover fermion configurations on an anisotropic $24^{3} \times 128$ lattice with comparable spatial lattice spacing and pion mass, i.e., with $a_{s}=0.123 \mathrm{fm}, \xi=3.5$, and $m_{\pi}=390 \mathrm{MeV}$. Again, we find that the SEB and variational calculations agree when a large smearing-sized interpolation field is included in the variation calculation. The Roper mass on this clover lattice is $\approx 280 \mathrm{MeV}$ higher than the value found on the previous lattice with overlap fermions at the same pion mass. It is clear that the difference is not due to the fitting algorithm.

To understand the definite difference between the two fermion actions, we examine the coupling of single-hadron interpolating fields to two hadron states. This explores the idea that the three-quark core couples to the higher Fock space $N \pi, N \eta$ and $N \pi \pi$ states which brings down the Roper mass from the quark model's uncoupled-three-quark-state value. To this end, we studied the would-be- $\eta \pi$ ghost state with the isovector scalar $\bar{\psi} \psi$ interpolation field in the quenched approximation. In this channel, it is well known that the would-be- $\eta \pi$ ghost state dominates at pion masses lower than $\approx 600 \mathrm{MeV}$ for the overlap fermion. This indicates that the one-hadron $\bar{\psi} \psi$ interpolation field will couple strongly to the physical $\eta \pi$ and $\eta^{\prime} \pi$ two-hadron states in the dynamical fermion setting.

We compare the $a_{0}$ correlators between the clover fermion and overlap fermion, both with HYP smearing. It is found that while the $\eta \pi$ ghost state shows up prominently with the overlap fermion on all three lattices at lattice spacings $a=0.12,0.09$ and $0.06 \mathrm{fm}$, the coupling to the ghost state are not as strong in the clover cases for $a=0.12,0.09 \mathrm{fm}$; the minima of their $a_{0}$ correlator which characterize the strength of the coupling to the $\eta \pi$ in the hairpin diagram are only half as strong as those of their corresponding overlap case. On the other hand, the $a_{0}$ correlators of the clover and overlap coincide within errors at $a=0.06 \mathrm{fm}$. We take this as an indication that chiral symmetry is restored for the clover fermion at this lattice spacing. It is not clear, at this stage, if this is due to the simple $\mathcal{O}\left(a^{2}\right)$ discretization error, or perhaps the chiral symmetry sets in abruptly near $a=0.06 \mathrm{fm}$. In any case, one would expect that the Roper will come down to the experimental value with the clover fermion at $a \leq 0.06 \mathrm{fm}$. We also compared Wilson and overlap fermions. The minima of the $a_{0}$ Wilson correlators are much shallower than those of the overlap for the above three lattices, reflecting the large $\mathcal{O}(a)$ error of the Wilson fermion. That the Wilson fermion is worse than the clover as far as the hairpin Z-graph is concerned is consistent with the fact that clover is chirally improved from the Wilson fermion.

We surmise that the different lattice results, described in this work, for the Roper state from the Wilson-type fermion versus overlap fermion and their associated chiral behaviors for the quenched ghost would-be- $\eta \pi$ state supports a resolution of the "Roper puzzle" due to $Z$-graph type chiral dynamics. This entails coupling to higher Fock-space components (e.g., $N \pi, N \pi \pi$ states) to induce the effective flavor-spin interaction between quarks prescribed in the chiral quark model, resulting in the parity-reversal pattern as observed in the excited states of $N, \Delta$ and $\Lambda$. This work is also consistent with the conclusion about the existence of higher Fock-space components in the Roper when experimental information on the nucleon to Roper transition form factors is examined [17].

In this study, we concentrate on comparing the Roper state calculated from the 3-quark interpolation field and try to reconcile the difference between the results from the clover fermion and the overlap fermion. Since the width of the Roper is small compared to its mass, we believe that the mass calculated from the 3-quark interpolation field should be close to the resonance mass from the $\pi N$ scattering, much like the case of the $\rho$ resonance from the $\pi \pi$ scattering. In the future, when the pion mass is close to the physical one, it would be important to carry out a lattice calculation which includes the $N \pi$ and $N \pi \pi$ channels to locate the pole position of the Roper and its width from the phase shift.

\section{ACKNOWLEDGMENTS}

We thank the RBC and UKQCD Collaborations for providing their DWF gauge configurations. This work is supported in part by the U.S. DOE Grant No. DESC0013065 and DOE Grant No. DE-AC05-06OR23177 which is within the framework of the TMD Topical Collaboration. A. A. is supported by National Science Foundation CAREER Grant No. PHY-1151648. A. A. 
and F. L. are supported by U.S. DOE Grant No. DE-FG0295ER40907. A. L. thanks the Institute for Nuclear Theory at the University of Washington for its kind hospitality and stimulating research environment. His research was supported in part by the INT's U.S. DOE Grant No. DE-FG0200ER41132. This research used resources of the Oak Ridge Leadership Computing Facility at the Oak Ridge National Laboratory, which is supported by the Office of Science of the U.S. Department of Energy under Contract No. DEAC05-00OR22725. This work used Stampede time under the Extreme Science and Engineering Discovery Environment (XSEDE), which is supported by National Science Foundation Grant No. ACI-1053575. We also thank the National Energy Research Scientific Computing Center (NERSC) for providing HPC resources that have contributed to the research results reported within this paper. We acknowledge the facilities of the USQCD Collaboration used for this research in part, which are funded by the Office of Science of the U.S. Department of Energy.
[1] K. F. Liu and C. W. Wong, Phys. Rev. D 28, 170 (1983).

[2] S. Capstick and N. Isgur, Phys. Rev. D 34, 2809 (1986).

[3] S. Capstick, Prog. Part. Nucl. Phys. 45, S241 (2000).

[4] K. F. Liu, J. S. Zhang, and G. R. E. Black, Phys. Rev. D 30, 2015 (1984).

[5] J. D. Breit and C. R. Nappi, Phys. Rev. Lett. 53, 889 (1984).

[6] U. B. Kaulfuss and U.-G. Meissner, Phys. Lett. 154B, 193 (1985).

[7] L. Glozman and D. Riska, Phys. Rep. 268, 263 (1996).

[8] K. F. Liu, S. J. Dong, T. Draper, D. Leinweber, J. H. Sloan, W. Wilcox, and R. M. Woloshyn, Phys. Rev. D 59, 112001 (1999).

[9] B. Julia-Diaz, T. S. H. Lee, A. Matsuyama, and T. Sato, Phys. Rev. C 76, 065201 (2007).

[10] H. Kamano, S. X. Nakamura, T. S. H. Lee, and T. Sato, Phys. Rev. C 81, 065207 (2010).

[11] N. Suzuki, B. Julia-Diaz, H. Kamano, T. S. H. Lee, A. Matsuyama, and T. Sato, Phys. Rev. Lett. 104, 042302 (2010).

[12] T. Barnes and F. Close, Phys. Lett. B 123, 89 (1983).

[13] Z. P. Li, Phys. Rev. D 44, 2841 (1991).

[14] C. E. Carlson and N. C. Mukhopadhyay, Phys. Rev. Lett. 67, 3745 (1991).

[15] O. Krehl, C. Hanhart, S. Krewald, and J. Speth, Phys. Rev. C 62, 025207 (2000).

[16] R. Jaffe and F. Wilczek, Eur. Phys. J. C 33, S38 (2004).

[17] V. D. Burkert and C. D. Roberts, Rev. Mod. Phys. 91, 011003 (2019).

[18] F. X. Lee (LHPC Collaboration), Nucl. Phys. B, Proc. Suppl. 94, 251 (2001).

[19] S. Sasaki, T. Blum, and S. Ohta, Phys. Rev. D 65, 074503 (2002).

[20] D. G. Richards, M. Gockeler, R. Horsley, D. Pleiter, P. E. L. Rakow, G. Schierholz, and C. M. Maynard (LHPCUKQCD, QCDSF Collaborations), Nucl. Phys. B, Proc. Suppl. 109A, 89 (2002).

[21] W. Melnitchouk, S. O. Bilson-Thompson, F. D. R. Bonnet, J. N. Hedditch, F. X. Lee, D. B. Leinweber, A. G. Williams, J. M. Zanotti, and J. B. Zhang, Phys. Rev. D 67, 114506 (2003).

[22] R. G. Edwards, U. M. Heller, and D. G. Richards (LHP Collaboration), Nucl. Phys. B, Proc. Suppl. 119, 305 (2003).
[23] K. Sasaki, S. Sasaki, and T. Hatsuda, Phys. Lett. B 623, 208 (2005).

[24] N. Mathur, Y. Chen, S. J. Dong, T. Draper, I. Horváth, F. X. Lee, K. F. Liu, and J. B. Zhang, Phys. Lett. B 605, 137 (2005).

[25] D. Brommel, P. Crompton, C. Gattringer, L. Ya. Glozman, C. B. Lang, S. Schaefer, and A. Schafer (Bern-GrazRegensburg Collaboration), Phys. Rev. D 69, 094513 (2004).

[26] S. Basak, R. G. Edwards, G. T. Fleming, J. Juge, A. C. Lichtl, C. Morningstar, D. G. Richards, I. Sato, and S. J. Wallace, arXiv:hep-lat/0609052.

[27] T. Burch, C. Gattringer, L. Ya. Glozman, C. Hagen, D. Hierl, C. B. Lang, and A. Schafer, Phys. Rev. D 74, 014504 (2006).

[28] B. G. Lasscock, J. N. Hedditch, W. Kamleh, D. B. Leinweber, W. Melnitchouk, A. G. Williams, and J. M. Zanotti, Phys. Rev. D 76, 054510 (2007).

[29] M. S. Mahbub, A. O. Cais, W. Kamleh, D. B. Leinweber, and A. G. Williams, Phys. Rev. D 82, 094504 (2010).

[30] M. S. Mahbub, W. Kamleh, D. B. Leinweber, P. J. Moran, and A. G. Williams, Phys. Lett. B 707, 389 (2012).

[31] M. S. Mahbub, W. Kamleh, D. B. Leinweber, P. J. Moran, and A. G. Williams, Phys. Rev. D 87, 094506 (2013).

[32] R. G. Edwards, J. J. Dudek, D. G. Richards, and S. J. Wallace, Phys. Rev. D 84, 074508 (2011).

[33] C. Alexandrou, T. Korzec, G. Koutsou, and T. Leontiou, Phys. Rev. D 89, 034502 (2014).

[34] G. P. Engel, C. B. Lang, D. Mohler, and A. Schfer (BGR Collaboration), Phys. Rev. D 87, 074504 (2013).

[35] K.-F. Liu, Y. Chen, M. Gong, R. Sufian, M. Sun, and A. Li, Proc. Sci., LATTICE2013 (2014) 507 [arXiv:1403.6847].

[36] K.-F. Liu, Int. J. Mod. Phys. E 26, 1740016 (2017).

[37] C. B. Lang, L. Leskovec, M. Padmanath, and S. Prelovsek, Phys. Rev. D 95, 014510 (2017).

[38] A. L. Kiratidis, W. Kamleh, D. B. Leinweber, Z.-W. Liu, F. M. Stokes, and A. W. Thomas, Proc. Sci., INPC2016 (2017) 269 [arXiv:1704.08816].

[39] M. Lujan, A. Alexandru, Y. Chen, T. Draper, W. Freeman, M. Gong, F. X. Lee, A. Li, K. F. Liu, and N. Mathur, Phys. Rev. D 86, 014501 (2012).

[40] Y. Chen, S.-J. Dong, T. Draper, I. Horvath, K.-F. Liu, N. Mathur, S. Tamhankar, C. Srinivasan, F. X. Lee, and J.-B. Zhang, arXiv:hep-lat/0405001. 
[41] N. Mathur, A. Alexandru, Y. Chen, S. J. Dong, T. Draper, I. Horváth, F. X. Lee, K. F. Liu, S. Tamhankar, and J. B. Zhang, Phys. Rev. D 76, 114505 (2007).

[42] Y. Chen, C. Liu, Y. Liu, J. Ma, and J. Zhang (CLQCD Collaboration), arXiv:hep-lat/0701021.

[43] J. J. Dudek, R. G. Edwards, and C. E. Thomas, Phys. Rev. D 86, 034031 (2012).

[44] C. Jung, C. Kelly, R. D. Mawhinney, and D. J. Murphy, Phys. Rev. D 97, 054503 (2018).

[45] T. Blum et al. (RBC, UKQCD Collaborations), Phys. Rev. D 93, 074505 (2016).

[46] Y. Chen, Mod. Phys. Lett. A 22, 583 (2007).

[47] D. S. Roberts, W. Kamleh, and D. B. Leinweber, Phys. Rev. D 89, 074501 (2014).

[48] M. Gong et al. (XQCD Collaboration), Phys. Rev. D 88, 014503 (2013).

[49] C. Michael, Nucl. Phys. B259, 58 (1985).

[50] M. Luscher and U. Wolff, Nucl. Phys. B339, 222 (1990).

[51] Y. Chen et al., Phys. Rev. D 73, 014516 (2006).

[52] B. Blossier, M. Della Morte, G. von Hippel, T. Mendes, and R. Sommer, J. High Energy Phys. 04 (2009) 094.

[53] D. S. Roberts, W. Kamleh, and D. B. Leinweber, Phys. Rev. D 89, 074501 (2014).

[54] J. Liang, Y.-B. Yang, K.-F. Liu, A. Alexandru, T. Draper, and R. S. Sufian, Phys. Rev. D 96, 034519 (2017).

[55] M. S. Mahbub, A. O. Cais, W. Kamleh, B. G. Lasscock, D. B. Leinweber, and A. G. Williams, Phys. Rev. D 80, 054507 (2009).
[56] M. S. Mahbub, W. Kamleh, D. B. Leinweber, A. O Cais, and A. G. Williams, Phys. Lett. B 693, 351 (2010).

[57] D. Guo, A. Alexandru, R. Molina, and M. Dring, Phys. Rev. D 94, 034501 (2016).

[58] A. Alexandru and R. Brett (private communication).

[59] D. J. Wilson, R. A. Briceno, J. J. Dudek, R. G. Edwards, and C. E. Thomas, Phys. Rev. D 92, 094502 (2015).

[60] I. G. Aznauryan and V. D. Burkert, Prog. Part. Nucl. Phys. 67, 1 (2012).

[61] W. A. Bardeen, A. Duncan, E. Eichten, N. Isgur, and H. Thacker, Phys. Rev. D 65, 014509 (2001).

[62] W. A. Bardeen, E. Eichten, and H. Thacker, Phys. Rev. D 69, 054502 (2004).

[63] S. Prelovsek and K. Orginos (RBC Collaboration), Nucl. Phys. B, Proc. Suppl. 119, 822 (2003).

[64] S. Prelovsek, C. Dawson, T. Izubuchi, K. Orginos, and A. Soni, Phys. Rev. D 70, 094503 (2004).

[65] E. Witten, Nucl. Phys. B156, 269 (1979).

[66] G. Veneziano, Nucl. Phys. B159, 213 (1979).

[67] L. Ya. Glozman, W. Plessas, K. Varga, and R. F. Wagenbrunn, Phys. Rev. D 58, 094030 (1998).

[68] M. Padmanath, C. B. Lang, L. Leskovec, and S. Prelovsek, EPJ Web Conf. 175, 05004 (2018).

[69] A. L. Kiratidis, W. Kamleh, D. B. Leinweber, Z.-W. Liu, F. M. Stokes, and A. W. Thomas, Phys. Rev. D 95, 074507 (2017).

[70] N. Isgur, Phys. Rev. D 61, 118501 (2000).

[71] K. F. Liu, S. J. Dong, T. Draper, J. H. Sloan, W. Wilcox, and R. M. Woloshyn, Phys. Rev. D 61, 118502 (2000). 